\title{
Lepton mixing under the lepton charge nonconservation, neutrino masses and oscillations and the "forbidden" decay $\mu^{-} \rightarrow e^{-}+\gamma$
}

\author{
Valery LYUBOSHITZ * \\ Joint Institute for Nuclear Research (Dubna, Russia) \\ E-mail: Valery.Lyuboshitz@jinr.ru

\section{Vladimir Lyuboshitz} \\ Joint Institute for Nuclear Research (Dubna, Russia)
}

The lepton-charge $\left(L_{e}, L_{\mu}, L_{\tau}\right.$ ) nonconserving interaction leads to the mixing of the electron, muon and tau neutrinos, which manifests itself in spatial oscillations of a neutrino beam, and also to the mixing of the electron, negative muon and tau lepton, which, in particular, may be the cause of the "forbidden" radiative decay of the negative muon into the electron and $\gamma$ quantum. Under the assumption that the nondiagonal elements of the mass matrices for neutrinos and ordinary leptons, connected with the lepton charge nonconservation, are the same, and by performing the joint analysis of the experimental data on neutrino oscillations and experimental restriction for the probability of the decay $\mu^{-} \rightarrow e^{-}+\gamma$ per unit time, the following estimate for the lower bound of neutrino mass has been obtained: $m^{(v)}>1.5 \frac{\mathrm{eV}}{c^{2}}$.

The European Physical Society Conference on High Energy Physics - EPS-HEP2013

18-24 July, 2013

Stockholm, Sweden

\footnotetext{
* Speaker
} 


\section{Mass matrix and states with definite masses for neutrinos and charged leptons}

Taking into account the $C P$ invariance ( $T$ invariance ), the mass matrix for the neutrino family should be symmetric and, due to hermiticity, real. It has the standard 3-row structure with the diagonal elements representing the masses of electron neutrino, muon neutrino and tau neutrino, whereas the nondiagonal elements $\left(m_{e \mu}^{(v)} \equiv m_{\mu e}^{(v)}, m_{e \tau}^{(v)} \equiv m_{\tau e}^{(v)}, m_{\mu \tau}^{(v)} \equiv m_{\tau \mu}^{(v)}\right)$ characterize the degree of lepton charge nonconservation. In doing so, the states with the definite lepton charge ("flavor") $\left|v_{e}\right\rangle,\left|v_{\mu}\right\rangle,\left|v_{\tau}\right\rangle$ are connected with the stationary states $\left|v_{1}\right\rangle,\left|v_{2}\right\rangle,\left|v_{3}\right\rangle$, being related with the definite masses $m_{1}, m_{2}, m_{3}$, by the unitary transformation and the respective unitary matrix $\hat{U}$ is real [1] . As a result, all the nondiagonal elements of the mass matrix can be expressed through the differences of masses of neutrino stationary states and the elements of the unitary matrix $\hat{U}$ ( the detailed consideration for $m_{e \mu}^{(v)}$ is given in [1] ) . Finally, using the representation of the mixing angles for the neutrino stationary states - which are formally analogous to the Maiani angles of quark mixing [2-5] - and assuming the masses of all the three stationary neutrinos to be approximately equal to each other [1] , we obtain the following formula for $m_{e \mu}^{(v)}[1]\left(m^{(v)}\right.$ is the common neutrino mass ) :

$$
\begin{aligned}
m_{e \mu}^{(v)} & =\frac{1}{4 m^{(v)}}\left[\left(\sin 2 \theta_{12} \cos \theta_{23} \cos \theta_{13}+\cos ^{2} \theta_{12} \sin \theta_{23} \sin 2 \theta_{13}\right)\left(m_{2}^{2}-m_{1}^{2}\right)+\right. \\
& \left.+\sin 2 \theta_{13} \sin \theta_{23}\left(m_{3}^{2}-m_{2}^{2}\right)\right] .
\end{aligned}
$$

For the family of leptons, taking into account the lepton-charge non-conserving interaction, the mass matrix is quite analogous to that for the neutrino family - its diagonal elements are equal to the masses of electron, negative muon and $\tau^{-}$lepton, whereas the nondiagonal elements $\left(M_{e \mu} \equiv M_{\mu e}, M_{e \tau} \equiv M_{\tau e}, M_{\mu \tau} \equiv M_{\tau \mu}\right)$ are negligibly small as compared with the electron mass $M_{e}$ and with all the differences of lepton masses.

Within the perturbation theory first-order approximation, the stationary states of leptons, related with definite masses, are the superpositions of states with different lepton charges [1] :

$\left|e^{\prime}\right\rangle=|e\rangle+\varepsilon_{\mu e}|\mu\rangle_{e}+\varepsilon_{\tau e}|\tau\rangle_{e},\left|\mu^{\prime}\right\rangle=|\mu\rangle+\varepsilon_{e \mu}|e\rangle_{\mu}+\varepsilon_{\tau \mu}|\tau\rangle_{\mu},\left|\tau^{\prime}\right\rangle=|\tau\rangle+\varepsilon_{e \tau}|e\rangle_{\tau}+\varepsilon_{\mu \tau}|\mu\rangle_{\tau}$

The masses of stationary states practically coincide with the masses of leptons ; meantime, the mixing coefficients in Eq. (2) $\left(\left|\varepsilon_{e \mu}\right|,\left|\varepsilon_{e \tau}\right|,\left|\varepsilon_{\mu \tau}\right|<<1\right)$ are expressed through the ratios of nondiagonal elements of the mass matrix to the differences of masses of respective leptons [1]

\section{Decay $\mu^{-} \rightarrow e^{-}+\gamma$ and estimate of the lower bound of neutrino mass}

Now let us estimate the probability of the "forbidden" radiative decay $\mu^{-} \rightarrow e^{-}+\gamma$ per unit time, assuming that this decay occurs on account of "admixture" of the state $|e\rangle_{\mu}$ with the electronic lepton number $L_{e}=1$ to the state of negative muon ( see Eq. (2) ). We may write : 


$$
W\left(\mu^{-} \rightarrow e^{-}+\gamma\right)=\left|\varepsilon_{e \mu}\right|^{2} W\left(|e\rangle_{\mu} \rightarrow e^{-}+\gamma\right) .
$$

In doing so, the probability $W\left(|e\rangle_{\mu} \rightarrow e^{-}+\gamma\right)$ may be calculated applying the standard guidelines of quantum electrodynamics [6] ( see [1] ) . Finally, we obtain the relation [1] :

$$
W\left(\mu^{-} \rightarrow e^{-}+\gamma\right)=W\left(\mu^{+} \rightarrow e^{+}+\gamma\right)=\left(\frac{M_{e \mu}}{M_{\mu}-M_{e}}\right)^{2} \frac{e^{2}}{2 \hbar c} \frac{M_{\mu} c^{2}}{\hbar} \approx \frac{M_{e \mu}^{2}}{2 M_{\mu}} \frac{e^{2}}{\hbar c} \frac{c^{2}}{\hbar} .
$$

According to the experimental data [7], $W\left(\mu^{-} \rightarrow e^{-}+\gamma\right)<1.2 \cdot 10^{-11} W\left(\mu^{-} \rightarrow e^{-}+\bar{v}_{e}+v_{\mu}\right)$ $\left(W\left(\mu^{-} \rightarrow e^{-}+\bar{v}_{e}+v_{\mu}\right)=0.455 \cdot 10^{6} \mathrm{sec}^{-1}\right)$. Thus, $W\left(\mu^{-} \rightarrow e^{-}+\gamma\right)<0.546 \cdot 10^{-5} \mathrm{sec}^{-1}$ and, due to Eqs. (3),(4), $\left|\varepsilon_{e \mu}\right|^{2}<0.936 \cdot 10^{-26},\left|M_{e \mu}\right|^{2}<1.032 \cdot 10^{-10} \mathrm{eV}^{2} / c^{4},\left|M_{e \mu}\right|<1.016 \cdot 10^{-5} \mathrm{eV} / \mathrm{c}^{2}$.

Further, let us make a natural assumption that the mixing of ordinary leptons $(e, \mu, \tau)$ and the mixing of neutrinos $\left(v_{e}, v_{\mu}, v_{\tau}\right)$ are conditioned by the same lepton-charge nonconserving interaction . Then the nondiagonal elements of mass matrices for the lepton family and for neutrinos should coincide : $M_{e \mu}=m_{e \mu}^{(v)}, M_{e \tau}=m_{e \tau}^{(v)}, M_{\mu \tau}=m_{\mu \tau}^{(v)}$, and we obtain - taking into account Eq. (1) for $m_{e \mu}^{(v)}-$ the following inequality for the neutrino mass $m^{(v)}$ [1] :

$$
\begin{gathered}
\left|M_{e \mu}\right|=\left|m_{e \mu}^{(v)}\right|=\frac{1}{4 m^{(v)}} \mid\left(\sin 2 \theta_{12} \cos \theta_{23} \cos \theta_{13}+\cos ^{2} \theta_{12} \sin \theta_{23} \sin 2 \theta_{13}\right)\left(m_{2}^{2}-m_{1}^{2}\right)+ \\
+\sin 2 \theta_{13} \sin \theta_{23}\left(m_{3}^{2}-m_{2}^{2}\right) \mid<1.016 \cdot 10^{-5} \mathrm{eV} / c^{2} .
\end{gathered}
$$

In accordance with the experimental data on neutrino oscillations [7] , assuming firstly that $\sin ^{2} 2 \theta_{12}=0.86\left(\theta_{12}=34^{\circ}\right), \sin ^{2} 2 \theta_{23}=0.92\left(\theta_{23}=36.8^{\circ}\right), \theta_{13}=0^{\circ},\left|m_{2}^{2}-m_{1}^{2}\right|=8 \cdot 10^{-5} \mathrm{eV} / c^{2}$, we find : $m^{(v)}>1.46 \mathrm{eV} / c^{2}[1]$. This value for the lower bound of neutrino mass is in agreement with the upper limit of antineutrino mass $\left(m^{(v)}<2.3 \mathrm{eV} / c^{2}[8], m^{(v)}<2.5 \mathrm{eV} / c^{2}\right.$ [9] $)$, derived within the study of electron spectrum in the tritium $\beta$-decay ( see also [7] ) .

Meantime, in case if $\left|M_{e \mu}\right| \neq\left|m_{e \mu}^{(v)}\right|$, the value for the lower bound of neutrino mass will change : $m^{(v)}>1.46|\eta| \mathrm{eV} / \mathrm{c}^{2}$, where $\eta=M_{e \mu} / m_{e \mu}^{(v)}$ is the ratio of nondiagonal elements of the mass matrices for leptons and neutrinos. If, in further experiments, the upper limit of the probability $W\left(\mu^{-} \rightarrow e^{-}+\gamma\right)$ will be reduced, this will testify to the fact that $|\eta|<1$.

Under another set of parameters, taking into account the latest data on the nonzero neutrino mixing angle $\theta_{13}: \sin ^{2} 2 \theta_{13}=0.095\left(\theta_{13}=9^{\circ}\right),\left|m_{3}^{2}-m_{2}^{2}\right|=3 \cdot 10^{-3} \mathrm{eV}^{2} / c^{4}$ [7] and the above values of $\theta_{12}, \theta_{23},\left|m_{2}^{2}-m_{1}^{2}\right|$ - we would obtain the estimate $m^{(v)}>(12.1 \div 15.1)|\eta| \mathrm{eV} / \mathrm{c}^{2}$, which may be in accordance with the presently known upper limits for the neutrino mass and probability of decay $\mu^{-} \rightarrow e^{-}+\gamma$ per 1 sec only at the ratios $\left|M_{e \mu} / m_{e \mu}^{(v)}\right|=|\eta|<\sim 10^{-1}$. 


\section{References}

1. V.L. Lyuboshitz, V.V. Lyuboshitz, Yad. Fiz. 76, 1003 (2013) [ Phys. At. Nucl. 76 , 947 (2013) ] .

2. B. Kayser (Particle Data Group), Phys. Lett. B 667, 163 (2008).

3. H. Goodman (Particle Data Group), Phys. Lett. B 667, 546 (2008).

4. L.B. Okun, Leptony i kwarki ( Leptons and Quarks ), Nauka, Moscow, 1990, Chapt. 15.

5. L. Maiani, Phys. Lett. B 62, 183 (1976).

6. V.B. Berestetsky, E.M. Lifshitz, L.P. Pitaevsky, Kvantovaya electrodinamika ( Quantum Electrodynamics ), Nauka, Moscow, 1983, §§ 43-45.

7. Review of Particle Physics, Phys. Lett. B 667 , 34, 36 (2008).

8. V.M. Lobashev et al., Phys. Lett. B 460, 227 (1999).

9. Ch. Kraus et al., Eur. Phys. J. C 40 ,447 (2005). 